\title{
Análise do uso e da ocupação da terra no município de Juiz de Fora dos anos de 1947 aos dias atuais ${ }^{1}$
}

\section{Analysis of the use and occupation of the land at the municipality of Juiz de Fora from the years of 1947 to the current days}

\author{
Gustavo Amaral Barbosa ${ }^{2}$ \\ Luciano Alves Soares Caramez $z^{3}$ \\ Ricardo T. Zaidan ${ }^{4}$
}

\section{DOI: https://doi.org/10.34019/2179-3700.2019.v19.29915}

\begin{abstract}
Resumo
Este trabalho traz uma apresentação do Projeto de Pesquisa de Iniciação Científica pela Universidade Federal de Juiz de Fora (UFJF) intitulado Análise espaço-temporal da evolução do uso, ocupação da terra no município de Juiz de Fora dos anos de 1947 aos dias atuais, com o propósito de resgatar do meio físico para o digital a Planta Cadastral de Juiz de Fora e o Plano de Remodelação e Extensão da cidade, ambos de 1947, elaborados pelo urbanista José Otacílio Saboya Ribeiro. Em síntese, apresenta-se a metodologia de digitalização dos produtos cartográficos citados e, posteriormente, as possibilidades de análise a partir do banco de dados obtidos e dos dados processados no software ArcGIS 10.

Palavras-chave: Planta Cadastral de Juiz de Fora; Plano de Remodelação e Extensãoda Cidade de Juiz de Fora; José Otacílio Saboya Ribeiro; produtos cartográficos.
\end{abstract}

\begin{abstract}
This paper presents a presentation of the Scientific Initiation Research Project by the Federal University of Juiz de Fora (UFJF), entitled Space-time analysis of land use evolution at the municipality of Juiz de Fora from the years 1947 to current days, with the purpose of recovering from the physical environment to digital the Juiz de Fora Cadastral Plan and the Remodeling and Extension Plan of the City of Juiz de Fora, both of 1947, prepared by the urban planner José OtacílioSaboya Ribeiro. In summary, it's demonstrated the methodology of digitalization of the cartographic products mentioned above and, later, the possibilities of analysis from the database obtained and data processed with the software ArcGIS 10.

Keywords: Juiz de Fora Cadastral Plan; Remodeling and Extension Plan of the City of Juiz de Fora; José Otacílio Saboya Ribeiro; cartographic products.

\footnotetext{
${ }^{1}$ Trabalho premiado no Seminário de Iniciação Científica da UFJF em 2018.

${ }^{2}$ Bolsista do Programa Institucional de Bolsas de Iniciação Científica da Universidade Federal de Juiz de Fora (PIBIC/UFJF).

${ }^{3}$ Bolsista do Programa Institucional de Bolsas de Iniciação Científica da Universidade Federal de Juiz de Fora (PIBIC/UFJF).

${ }^{4}$ Professor orientador da Bolsa de Iniciação Científica. Endereço profissional: Universidade Federal de Juiz de Fora - LGA (sala: B-III-03)/Departamento de Geociências/ICH/UFJF. Rua José Lourenço Kelmer, s/n Campus Universitário. Bairro São Pedro - CEP: 36036-900 - Juiz de Fora - MG, Brasil. E-mail:
} ricardo.zaidan@ufjf.edu.br.
\end{abstract}




\section{INTRODUÇÃO}

Duas datas marcantes presentes no brasão da cidade de Juiz de Fora (MG) remetem a uma história rica e peculiar: 1701 e 1850.A história da cidade começa, segundo Oliveira (1966), em 1701 com o decreto da Coroa Portuguesa que incumbiu à Garcia Rodrigues Paes a abertura do Caminho Novo, em 13 de janeiro de 1698 (OLIVEIRA, 1975). Outra data marcante para a história de Juiz de Fora foi a elevação do povoado à condição de vila, em 1850.

Desde esses acontecimentos, Juiz de Fora veio sofrendo grandes transformações, sobretudo na transição do modo de vida rural para o urbano. A cidade foi regida por códigos de posturas de 1853 e 1858 (já em condição de município), ambas trazendo em seus artigos a delimitação territorial do que se encontrava na época, no caso Vila de Santo Antônio do Paraibuna e Cidade do Paraibuna, respectivamente.

Doravante, com a promulgação dos códigos de posturas de caráter punitivo e delimitando o território da vila e da cidade,surgiram planejamentos urbanos preocupadoscom a urbanidade, os quais levaram à aprovação, em 18 de julho de 1860, da elaboração da primeira planta da Cidade do Paraibuna, pelo engenheiro Gustavo Dodt (OLIVEIRA, 1975). Trata-se de uma planta planimétrica, contendo apenas arruamentos e algumas edificações, que gerou controvérsia na época pela não fidelidade à condição que se encontrava a cidade. Outras plantasconhecidas da cidade de Juiz de Fora datam de 1883, elaborada por Uchôa Cavalcantti; e 1884, elaborada, segundo Passaglia (1982), em 23 de janeiro por Miguel Antônio Lallemond.

Além dessas, outra, em caráter singular, ou seja, planialtimétrica, foi elaborada pelo urbanista José Otacílio Saboya Ribeiro junto ao Instituto Geodésico Brasileiro: uma Planta Cadastral de Juiz de Fora (PCJF) que, segundo Machado (2016), foi providenciada em termos da elaboração de um projeto que se assemelha a um plano diretor para a remodelação e extensão da cidade, de 1947.

É importante destacar que o Plano de Remodelação e Extensão da Cidade de Juiz de Fora (PREJF) não originou artigos detalhando os procedimentos adotados para sua elaboração, voltado exclusivamente para Juiz de Fora.

Portanto, observa-se a ausência de trabalhos realizados para a cidade a partir da PCJF e do PREF. Este artigo surgiu como ensaio do projeto de iniciação científica Análise espaço-temporal da evolução do uso, ocupação da terra no município de Juiz de Fora dos 


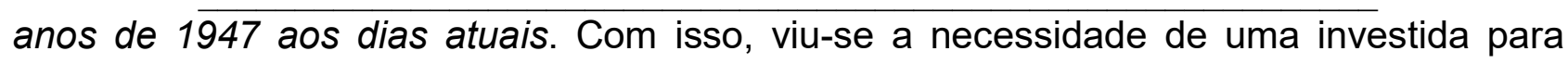
iniciar os estudos históricos e principalmente geográficos sobre os produtos cartográficos gerados pelo urbanista Saboya Ribeiro. Cabe destacar que a ênfase deste artigo são os procedimentos metodológicos adotados para a transformação do meio físico para o meio digital, da PCJF e do PREJF, e posteriormente, como resultados parciais, a elaboração de um mapa de evoluções e projeções da estrutura viária urbana de parte da região central e adjacências de Juiz de Fora para os anos de 1947 e 2007, como preliminar dos produtos cartográficos obtidos com a metodologia que será descrita.

\section{METODOLOGIA}

O procedimento metodológico primeiramente se baseou na pesquisa documental dos produtos cartográficos, a PCJF e o PREJF, totalizando 57 folhas na escala de 1:1000, para ambos. Logo, a metodologia obedece às etapas descritas a seguir.

\subsection{Etapa 1 - Transformação do produto cartográfico (físico) para o meio digital}

Para a PCJF, a etapa 1 consistiu em fotografar as folhas através de uma câmera fotográfica modelo Nikon D3100, resolução máxima de 14,2 megapixels, de lente $18 \mathrm{~mm}$ $\times 55 \mathrm{~mm}$, suspensa por uma haste (Figura 1 ), e editar as imagens.

Figura 1 -Sistema elaborado para realização das fotografias obtidas no software de edição de imagem Adobe Photoshop

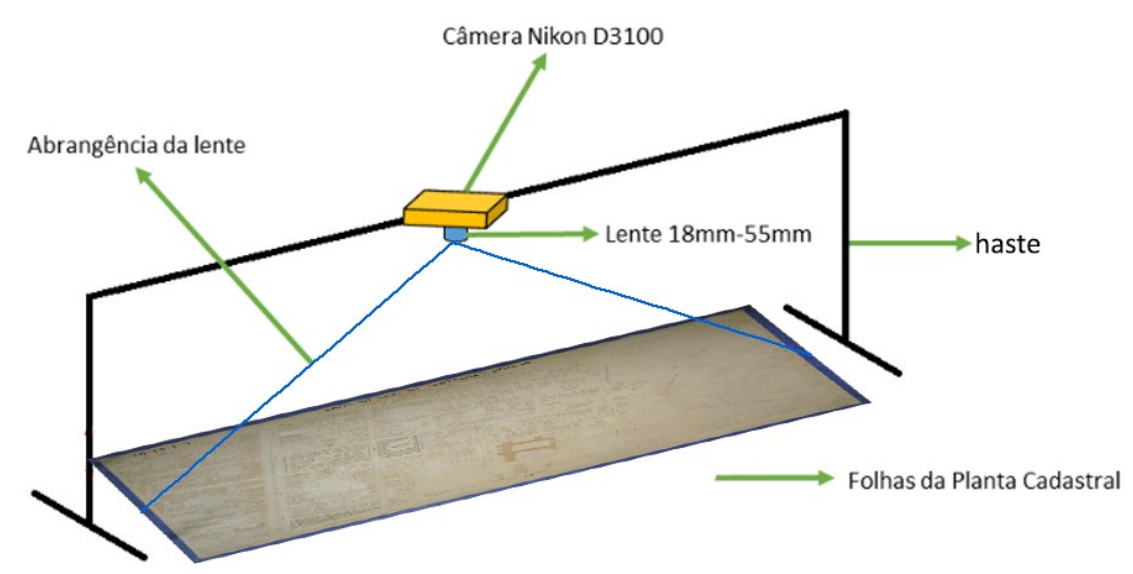

Fonte: Elaboração própria, 2018.

A metodologia aplicada para a digitalização da PCJF surgiu da necessidade de resolução de problemas técnicos. Para preservar as folhas da PCJF, cada folha foi colada 
em umMedium Density Fiberboard (MDF). Logo, com a espessura alterada das plantas, não foi possível realizar a digitalização via scanner, em razão de o espaço de alimentação ter altura inferior à espessura das pranchas (folha $+\mathrm{MDF}$ ), assim tornou-se necessário o uso da fotografia. Para obtenção digital do Plano de Remodelação e Extensão da Cidade de Juiz de Fora utilizou-se o scanner A0 (Figura 3).

A correção geométrica foi realizadaao aplicar o atalho Ctrl+Shift+R(Windows) noAdobe Photoshop, que realiza a correção da distorção; esse recurso é feito de maneira automática caso o software já estiver equipado com o plug-in que fornece as informações da câmera e detecta a distorção geométrica padrão da lente utilizada - tambémhá a maneira manual, consistindo em abrir a ferramenta Filtro/Correção de lente (Figura 2).

Figura 2 - Procedimento para realizar a correção geométrica da lente $18 \mathrm{~mm} \times 55 \mathrm{~mm}$

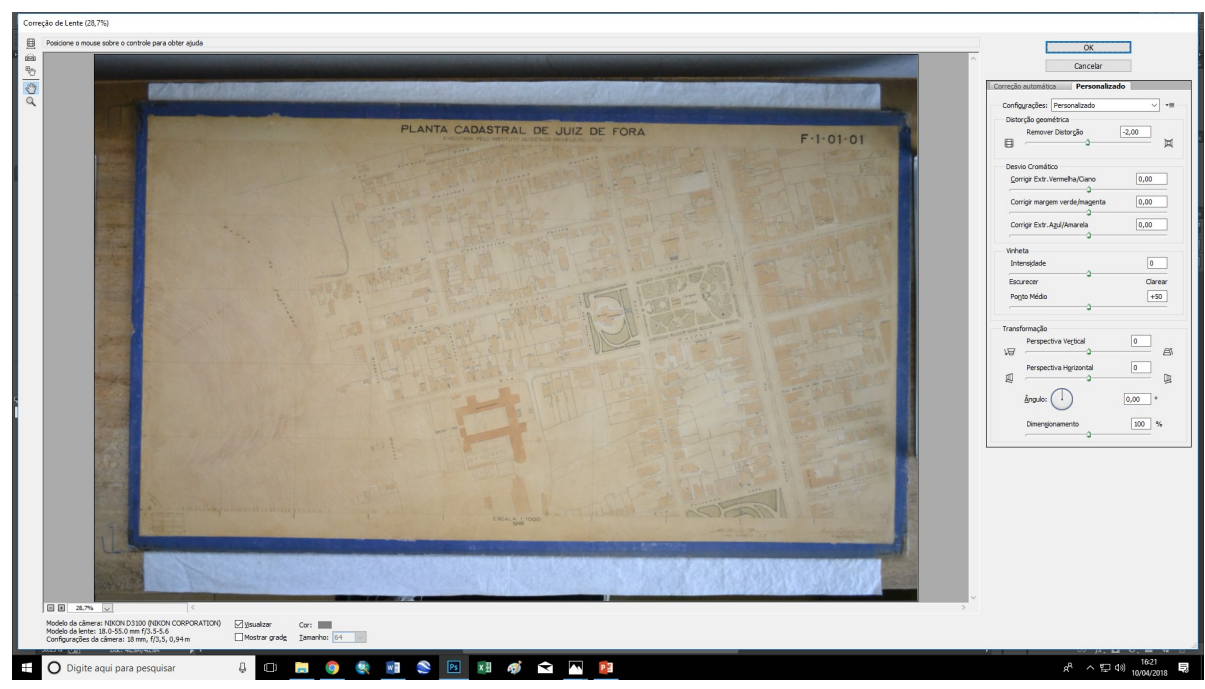

Fonte: elaboração própria.

Após a correção geométrica, outro procedimento adotado foi cortar as partes periféricas e nivelar a imagem para focar somente na planta. Para isso usou-se o atalho Ctrl+T e, por fim, para cortar as partes periféricas, usou-se a ferramenta Corte. 
Figura 3 - Folhas da PCJF e do PREJF

Folha F-1-01-01 da PCJF Digitalizada

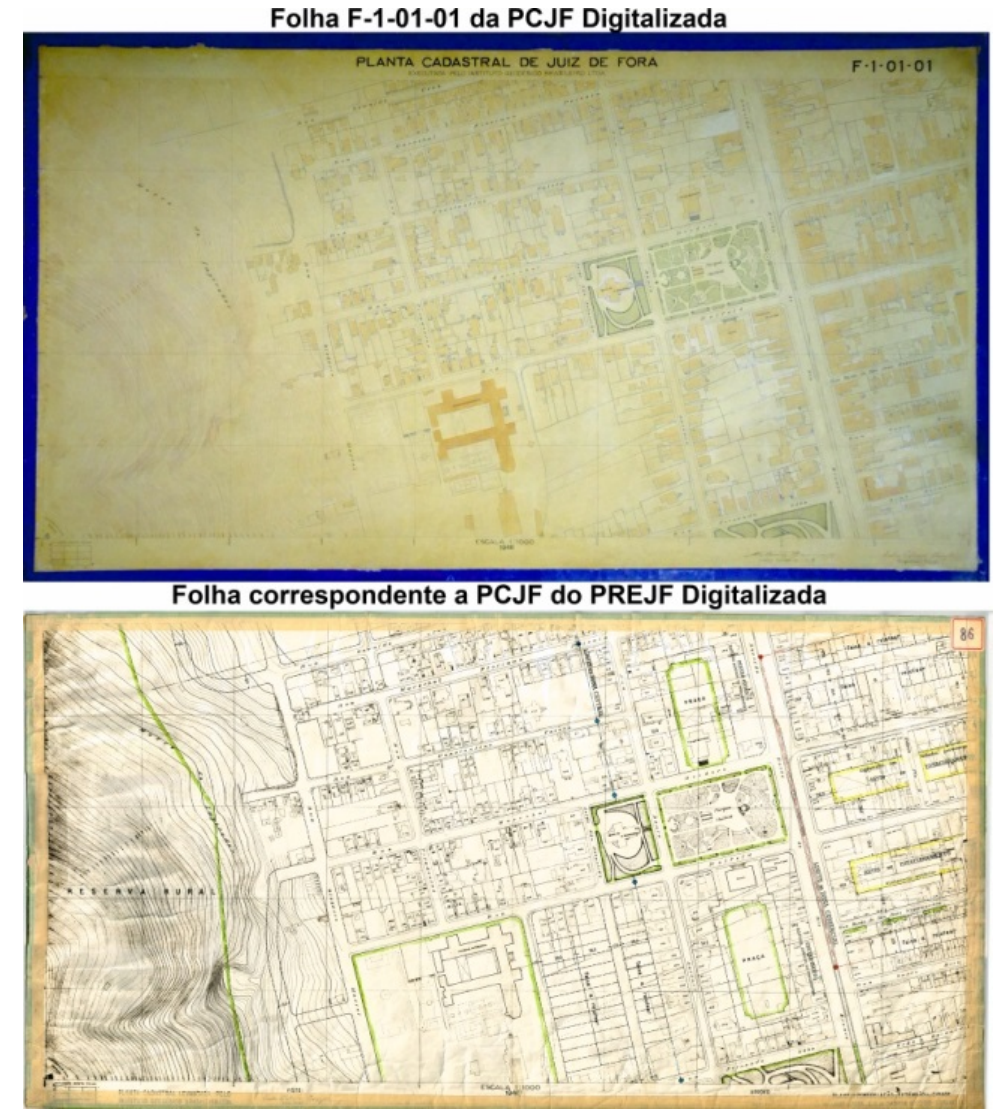

Fonte: Arquivo Histórico de Juiz de Fora.

\subsection{Etapa 2 - Execução do produto cartográfico final}

Esta etapa consistiu na elaboração de um mapa de evolução e projeções da estrutura viária urbana a partir da obtenção do banco de dados discriminados na etapa anterior. Para a elaboração desse mapa, utilizou-se o software ArcGIS 10; adicionou-se o levantamento aerofotogramétrico de 2007 (raster), as vias urbanas (shapefile) de Juiz de Fora e as folhas da PCJF e as do PREJF correspondentes (Tabela 1).

Tabela 1 - Folhas da PCJF e folhas do PREJF correspondentes

\begin{tabular}{c|c|c|c|c|c|c}
\hline PCJF & F-1-01-01 & F-1-01-01 & F-1-01-03 & F-2-01-01 & F-2-01-02 & F-2-01-03 \\
PREJF & 86 & 87 & 88 & 96 & 97 & 98 \\
\hline
\end{tabular}

Fonte: Contagem realizada pelo autor.

A visualização das estruturas viárias de 1947 e as projeções de ruas foram realizadas pelo georreferenciamento do PREJF em relação ao levantamento aerofotogramétrico de Juiz de Fora de 2007, com sistema de referência South American 
Datum 69, zona 23 Sul. A PCJF serviu como base para a referência de estruturas viárias inexistentes em 1947, ou seja, na PCJF não havia as projeções que se encontram no PREJF. Com isso, para as ruas projetadas foi criado um vetor shapefile para essa categoria; para as ruas que foram projetadas e concretizadas utilizou-se a ferramenta Editor de feição, como Fernandes (2016) utilizou em seu trabalho.

A partir da metodologia adotada, uma sistematização dos produtos cartográficos antigos propiciou uma análise fundamental encontrada no escopo do geoprocessamento denominado sobreposição, função que possibilita uma análise temporal.A sobreposição é importante pelo fato de a maioria das aplicações de informação geográfica integrar dados de diferentes origens (MIRANDA, 2005).

\section{RESULTADOS}

Juiz de Fora a partir da abertura da Avenida Barão do Rio Branco, idealizada e executada pelo engenheiro Henrique Fernando Halfeld, através da confirmação do contrato apresentada pela Lei ProvincialN 81, de 13 de abril de 1837, “Art. Único. É approvado o contracto feito pelo Governo com o Engenheiro Fernando Halfeld em data de 13 de maio de 1836" (MINAS GERAIS, 1837).

Juiz de Fora recebeu intensas modificações com a inserção ativa do ser humano na paisagem, não somente na questão da infraestrutura, mas também em seu valor simbólico (SANTOS, 2004 apud BARBOSA, 2017), principalmente depois de 1940, em que Juiz de Fora sofreu com um evento que mudaria a perspectiva do ordenamento territorial da cidade. Como afirmam Machado et al.(2016), entre os dias 22 e 26 de dezembro de 1940 tem lugar o acontecimento que mudaria definitivamente a história da cidade e de seu rio: ocorria a maior, mais violenta e mais prolongada enchente do rio Paraibuna.

Hoje, o que abriga parte da quadra central da cidade já pertenceu ao Juiz de Fora (margem esquerda do rio Paraibuna), juiz cuja titulação leva o nome da cidade. Posteriormente, a região, mais edificada, com maior estrutura viária e estabelecimentos que oferecem serviços (margem direita do rio Paraibuna), pertenceu a Antônio Dias Tostes, um dos personagens mais importantes da cidade; fazendeiro e produtor de café, dos 160 moradores de suas terras, 148 eram escravos (CEDEPLAR, 2017).

Partindo do princípio de acumulação primitiva de capital pela economia cafeeira, a região que hoje se conhece como o "centro", tornou-se um local valorizado e de prestígio, 
oportunizado principalmente por Henrique Halfeld, casado com uma das filhas de Antônio Dias Tostes, Cândida Maria Carlota, que, após o casamento, tornou-se dono de parte das terras. Com a construção da Estrada do Paraibuna, o ordenamento territorial acompanhou o novo eixo da estrada, hoje conhecida como Avenida Rio Branco, na parte mais plana da cidade, atraindo investidores do café na área ao longo da estrada/avenida.

A partir da elaboração do mapa "Evoluções e projeções da estrutura viária urbana da região central de Juiz de Fora" (Figura 4), iniciou-se um estudo preliminar das propostas feitas por Saboya Ribeiro no PREJF de 1947 e como elas aparecem em 2007. O recorte encontrado no mapa traz parte da região central e locais adjacentes, elucidando seu adensamento rodoviário.

Figura 4 -Evoluções e projeções da estrutura viária urbana da região central de Juiz de Fora (MG) - 1947/2007

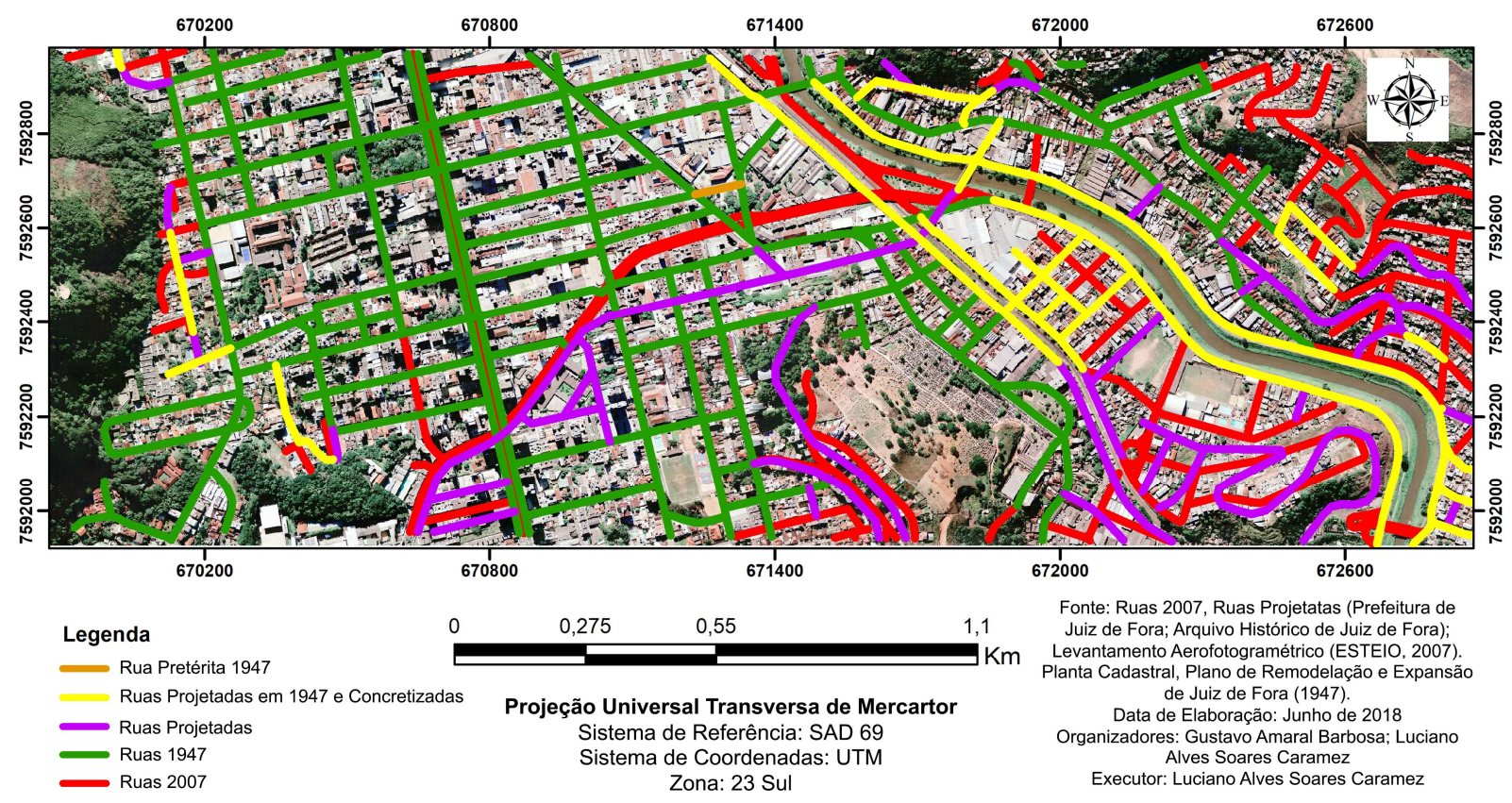

Fonte: Elaboração própria.

Logo, pelas observações feitas no PREJF, Saboya Ribeiro trazia propostas de reloteamentos para a abertura de ruas. Por conseguinte, notaram-se vias urbanas existentes em 1947 e também presentes em 2007 (verde), ruas projetadas em 1947 que não se concretizaram (roxo), ruas projetadas que se concretizaram (amarelo), ruas que existiam em 1947, mas deixaram de existir (bege) e ruas que existem em 2007, mas que 
não existiam em 1947 (vermelho), como mostra a Figura 4.

Com uma análise mais profunda a partir das projeções feitas por Saboya Ribeiro, verifica-se a nordeste da área de estudo um vetor de expansão urbana (margem esquerda do rio Paraibuna), um contrassenso, uma vez que o processo inicial de ocupação da cidade se deu pela margem esquerda do rio Paraibuna devido à abertura do Caminho Novo por Garcia Rodrigues Paes Leme, membro de uma família tradicional de bandeirantes (STEHLING, 1979). Com o desbravamento feito pelo bandeirante citado anteriormente, passou por uma região onde hoje se localizam os bairros Santo Antônio e Retiro, em Juiz de Fora, posteriormente assentaram-se sesmarias.

Próximo do que seria Juiz de Fora, o bandeirante passou rumo à montante, pela margem esquerda do Rio Paraibuna, onde hoje se localizam os bairros Retiro e Santo Antônio, a Avenida Sete de Setembro [...]. (STEHLING, 1979, p.102).

Com a abertura da Avenida Barão do Rio Branco a partir de 1837, o núcleo urbano passou para a margem direita do rio, assim, ruas planejadas e concretizadas remetem à realocação da estrutura urbana para a margem esquerda que, de acordo com os aspectos históricos, a ocupação populacional da região (Juiz de Fora) com a abertura do caminho novo na margem esquerda do rio Paraibuna, aconteceram a partir do morro da boiada.

Outra observação pertinente é sobre a rua paralela à Avenida Barão do Rio Branco, de suma importância para a logística da área central de Juiz de Fora, a Rua Santo Antônio. Pelo levantamento aerofotogramétrico de 2007, constatou-se um prolongamento desta rua até a Avenida Independência, atual Avenida Itamar Franco (uma importante via de ligação entre o Bairro São Pedro e o centro da cidade), em relação a 1947. Em contraponto de outras vias que foram planejadas, a Rua Santo Antônio não se enquadra na perspectiva do planejamento feito por Saboya Ribeiro.

\section{CONSIDERAÇÕES FINAIS}

O estudo realizado até o momento fez-se necessário para incentivar e enriquecer todos os trabalhos já feitos e os que virão pela frente. A adoção da metodologia de digitalização de produtos cartográficos antigos mencionada aqui, sobretudo da cidade de Juiz de Fora, abriu possibilidades para o levantamento e a sistematização de outros produtos cartográficos antigos da cidade, como as plantas de Gustavo Dodt e Miguel Antônio Lallemond.

Estudar os produtos cartográficos antigos possibilita análises futuras, inclusive para 
o meio urbano, que é o que envolve o trabalho comentado aqui; análises no âmbito das políticas públicas e do ordenamento territorial, propriamente dito. Ademais, este estudo rememora a história de Juiz de Fora, proporcionando valorização e preservação da reminiscência da cidade.

\section{AGRADECIMENTOS}

Agradecemos aos professores envolvidos nesta pesquisa, os quais foram tão importantes para a realização dos estudos: Prof ${ }^{a}$. Dra . Ana Aparecida Barbosa Pereira, Dr. Pedro José de Oliveira Machado e, em especial, ao professor orientador Dr. Ricardo Tavares Zaidan, pelos ensinamentos, pela paciência e pela confiança ao longo de todo o tempo de pesquisa.

Agradecemos também a toda a equipe do Arquivo Histórico da Prefeitura de Juiz de Fora, que sempre nos abraçou e disponibilizou o material cartográfico indispensável para nossa pesquisa.

Por fim, deixamos uma gratulação à comissão avaliadora do Seminário de Iniciação Científica do ano de 2018, que acreditou em nosso trabalho e nos proporcionou esse momento mágico.

\section{REFERÊNCIAS}

BARBOSA, Y. A. Pequena geografia histórica de Juiz de Fora: o processo urbano do Caminho Novo ao início do século XX. Juiz de Fora: FUNALFA, 2017.

CEDEPLAR, UFMG. Lista Nominativa de Santo Antônio de Juiz de Fora, 1831. Disponível em:

$<$ http://www.poplin.cedeplar.ufmg.br/principal.php?t=true\&popline=listaNominativa\&d=102 06>. Acesso em: mar. 2017.

FERNANDES, B. J. Evolução temporal e espacial do uso, ocupação e cobertura da terra na bacia hidrográfica do córrego Tapera (Juiz de Fora-MG). Revista de Geografia PPGEO/UFJF, Juiz de Fora, v. 6, n. 4, p. 377-386, 2016.

INSTITUTO DE PESQUISAS TECNOLÓGICAS (IPT). Curso de capacitação, mapeamento e gerenciamento de risco. Brasília DF: 2006. PREFEITURA DE JUIZ DE FORA. Plano Diretor de Desenvolvimento de Juiz de Fora - Diagnóstico. Juiz de Fora, 2004.

JUIZ DE FORA (Município). Plano diretor de desenvolvimento de Juiz de Fora Diagnóstico. Juiz de Fora, 2004.

MACHADO, P. J. O. Urbanização e modificações no córrego Independência, Juiz de Fora 
- MG. Cadernos do Núcleo de Análises Urbanas, v. 9, n. 1, p. 135-154, 2016.

MACHADO, P. J. O. et al. Juiz de Fora e a urbanização do Paraibuna. Revista de Geografia - PPGEO/UFJF, Juiz de Fora, v. 6, n. 1, p. 23-34, jan.-jun. 2016.

MINAS GERAIS (Estado). Lei Provincial $n^{\circ} 81$, de 13 de abril de 1837. Carta de Lei, que aprova o contrato feito pelo Governo com o Engenheiro Fernando Halfeld. Ouro Preto: Tipografia do Universal, 1837. p. 87-88. T. 3. Parte 1.

MIRANDA, J. I. Fundamentos de sistemas de informações geográficas. Brasília, DF: Embrapa Informação Tecnológica, 2005. 425 p.

OLIVEIRA, Paulino. História de Juiz de Fora. Juiz de Fora: Editora da UFJF, 1966.

OLIVEIRA, P. de. Efemérides juizforanas. Juiz de Fora: Ed. Universidade Federal de Juiz de Fora, 1975.

PASSAGLIA, L. A. do P. A preservação do patrimônio histórico de Juiz de Fora: medidas iniciais. Juiz de Fora: Prefeitura de Juiz de Fora, 1982.

STEHLING, L. J. A Companhia União e Indústria e os alemães. Juiz de Fora: Prefeitura Municipal de Juiz de Fora; FUNALFA, 1979. 